\title{
Textile Sensor Based Exo-Skeleton Suits for the Disabled
}

Sriram P. R, Muthu Manikandan M, Nithin Ayyappaa, Achuthan Ram

Sri Venkateswara College of Engineering

India

sriram.pr97@gmail.com

muthu.manikandan.m@gmail.com

ayyappannithin006@gmail.com

achyuthan1000@gmail.com

ABSTRACT: The exoskeleton suit is designed to make a person who is completely or partially paralyzed below the waist line capable of walking, running with minimal effort. This suit is mainly focused on people suffering from Paraplegia. The special ability of the following suit is that it does not make the person bulky rather it retains the natural formation and structure of a human. The following suit is based on textile based sensors that determine the movement and motion of the forearms. The advanced sensors placed in the forearm calculate the pattern and swinging motion of the forearm and formulates the action. The calculated motion of the forearm provides a gesture based input for the control system. Thus it provides a quicker response time for the manipulators to act according to the scenario. The suit consists of lithium ion battery inside a backpack which powers the manipulators. A control system is further initiated to the actuators placed in thigh, knee, ankle of the leg. The cutting-edge technology will be able to improve the muscle movement in the legs and help to permanently resolve the issue instead of relying on the use of wheel chairs for the rest of their lives.

Keywords: Exoskeleton Suit, Physically challenged, Intelligent control

DOI: $10.6025 / \mathrm{jdp} / 2018 / 8 / 3 / 89-94$

Received: 11 May 2018, Revised 18 June 2018, Accepted 27 June 2018

(C) 2018 DLINE. All Rights Reserved

\section{Introduction}

Paraplegia is an impairment in motor or sensory functions of the lower extremities. It is usually caused by spinal chord injuries or a congenital condition that affects the neural elements of the spinal canal. The American spinal injury association (ASIA) classifies the above condition to be extremely severe. This suit is designed to help the disabled people capable of performing their everyday activities in ease. The suit is designed using ultra-light aluminum alloys which provide maximum ease to the user. Self balancing technologies have been implemented to achieve stability. There are textile based sensors which are present in the sleeve of the shirt which the user wears on. These sensors are the new technology that we have implemented to the already 
existing exoskeleton technology. These sensors calculate the angle of the arm bending and processes the required criteria to provide the required speed for the suit to perform with. It boasts of high flexibility and rigidity. The suit consists of a lithium -ion battery producing power for the control system. The backpack also comprises of the gearbox which regulates the speed in which the suit operates. This is further connected to the manipulators, actuators and the sensors in the arm. The objective of the suit is to provide maximum efficiency and comfort to the user.

\section{Construction}

\subsection{Limb Support}

This is categorized as the functioning region. Aluminum alloy based limb support is designed using $C A D$ software and manufactured. This limb support consists of three parts, they are present in the thigh region, limb region and the ankle region and are named $L 1, L 2, L 3$ respectively. These are further connected with two pneumatic drive system actuators situated in the hip-thigh joint, limb joint and ankle joint named $A, B, C$ and $D$ respectively. This followed by a gear box connected with dc motors. The following is similar in both the legs.

\subsection{Backpack}

This is categorized as the power house and the processing unit of the suit. It consists of a lithiumion battery powering the TM4C1294NCPDT controller inside the backpack. It also comprises of a three geared gear box. This ensures three various speeds for movement- walking, fast walking and running. The back back is designed in a sleek and slim way to ensure that the suit does not become bulky. The battery provides sufficient power for the user to cover a maximum distance of 13,000 to 14,000 steps while walking and 4,000 to 8,000 steps while running, depending on the speed. The average person walks nearly 7,000 to 12,000 steps daily. Hence the following suit is sufficient enough for the user to carry out his day to day actions.

\subsection{Backpack}

A textile pressure sensor for muscle activity and motion detection equipped in the forearm of the disabled person to track the dynamic movement of the person. Example: Running, walking, climbing. A force pressure sensor is also equipped in the bottom of the heel in the suit to detect if the movement is continuous. A dedicated switch is found in the suit which helps the user to switch between walking and staying still. This can prevent unwanted movement while performing normal activities.

\section{Working}

\subsection{Textile Sensors}

The textile pressure sensor consists of a basic three layer structure forming a capacitance with a pressure sensing nonconducting dielectric. The sensor has been used to detect the activity of the muscles of the upper arm. When the muscle is contracted, it thickens and therefore the pressure of the muscle on the stretched band increases. This principle of the sensor allows the suit to determine the required motion of the person. Whether to walk, run or climb stairs. Not only the motion of the arm can be detected, but also the activity of the biceps and triceps can be measured accurately.

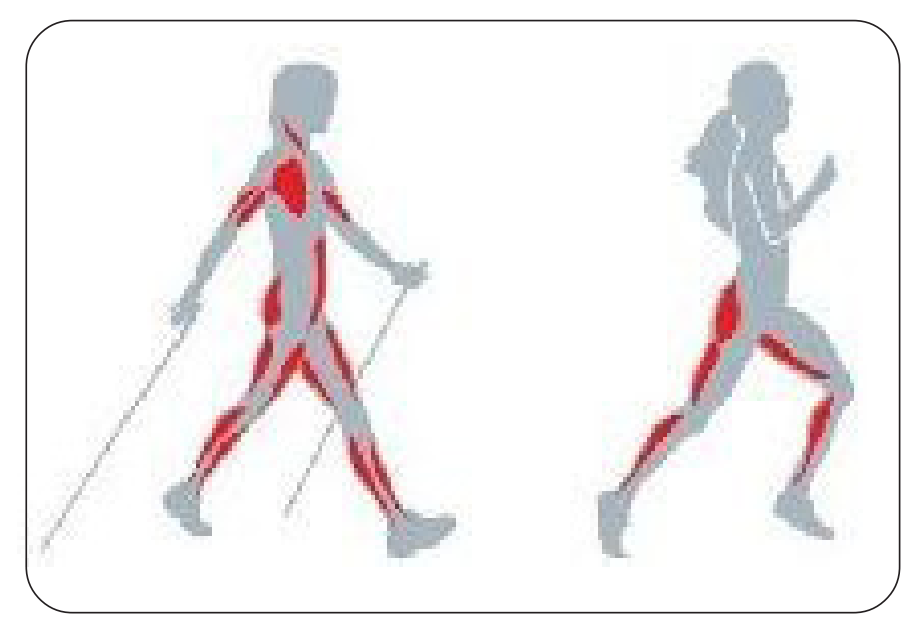

Figure 1. Walking, running 


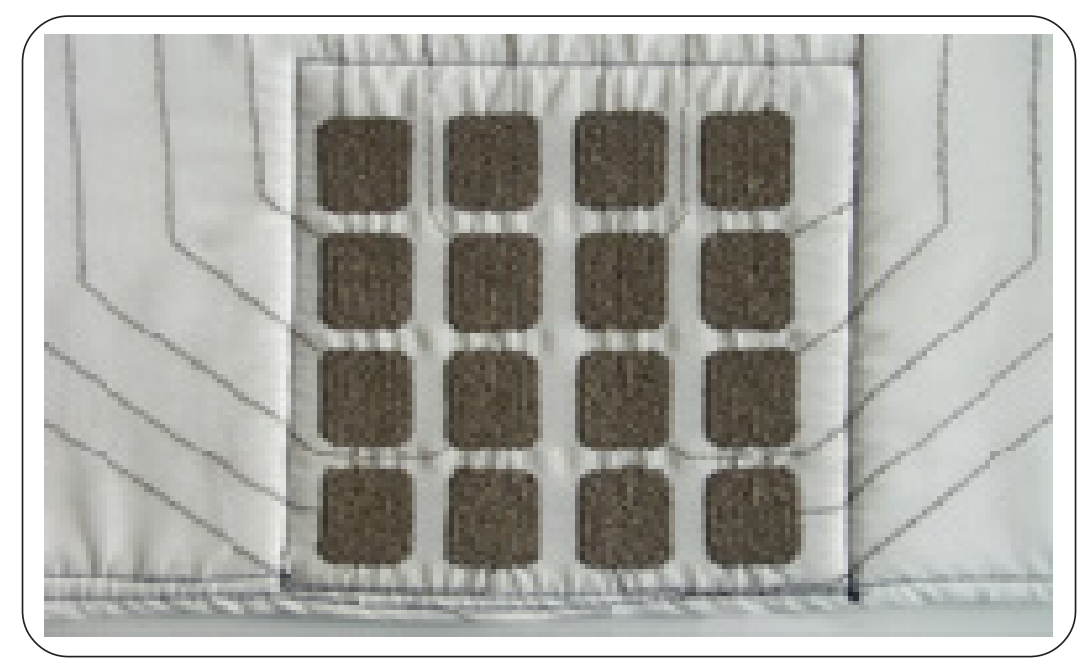

Figure 2. Textile pressure sensor with 16 sensing elements embroidered with conductive yarn

These accurate readings help us to figure out the exact angle in which the forearm is swung during a movement. In brief description we understand that when the human tends to walk the forearm is swung at an angle between 180 degrees -90 degrees. Similarly, during running, the forearm is swung at an angle between 90 degrees and 270 degrees. From the above context, we discover the dynamic motion of the forearm differentiating walking and running. The sensors located in the forearm identifies such change in the angle and sends the information to the micro-controller system. Another interesting notion on human body motion is that, the human hand is first initiated as a gesture and the opposite leg is swung in parallel, completing a movement.

So basically the movement of the suit basically depends on 3 major aspects.

- The angle of the forearm swinging movement

- The hand which swings

- Activity of biceps and triceps

This information is fed into the control system.

\section{Control System}

Control system is the place where all the analytical operations takes place and all the operations to be carried out are calculated here. This control system is embedded into the the backpack of the suit and along with the gearbox and the battery. TM4C1294NCPDT is the primary micro controller used in the following suit. This is the main control unit of the the exoskeleton. This is the main functioning unit of the suit. The control system is present in the backpack along with the battery and the gear box unit. The control system facilitates total control of the suit by regulating the speed of the suit by feeding the output to the gear box. This is the brain of the suit. All functions are carried out in this block. Self balancing of the suit is achieved using stabilising mechanism using the control system.

The left brain controls the right limbs whereas the right brain controls the left limbs. When we walk, there is continuous synchronization between the arms and the legs. Whenever right leg is put forward, the left arm swings in front and vice versa. Keeping this principle in mind, the exoskeleton suit is designed. The Figure describes about the control loop.

In the figure, is the body of person who is paralyzed from the waist line because he had an injury. So, to deal with this problem a textile sensor is fixed rigidly on both the arms. When left hand swings in front, the angle will decrease and reach a threshold point. This threshold point is that angle at which the arm swing angle is minimum. When a person walks his arms move in a specific manner. The textile sensors are used to calculate the angle of the arms and sends the information to the computing 


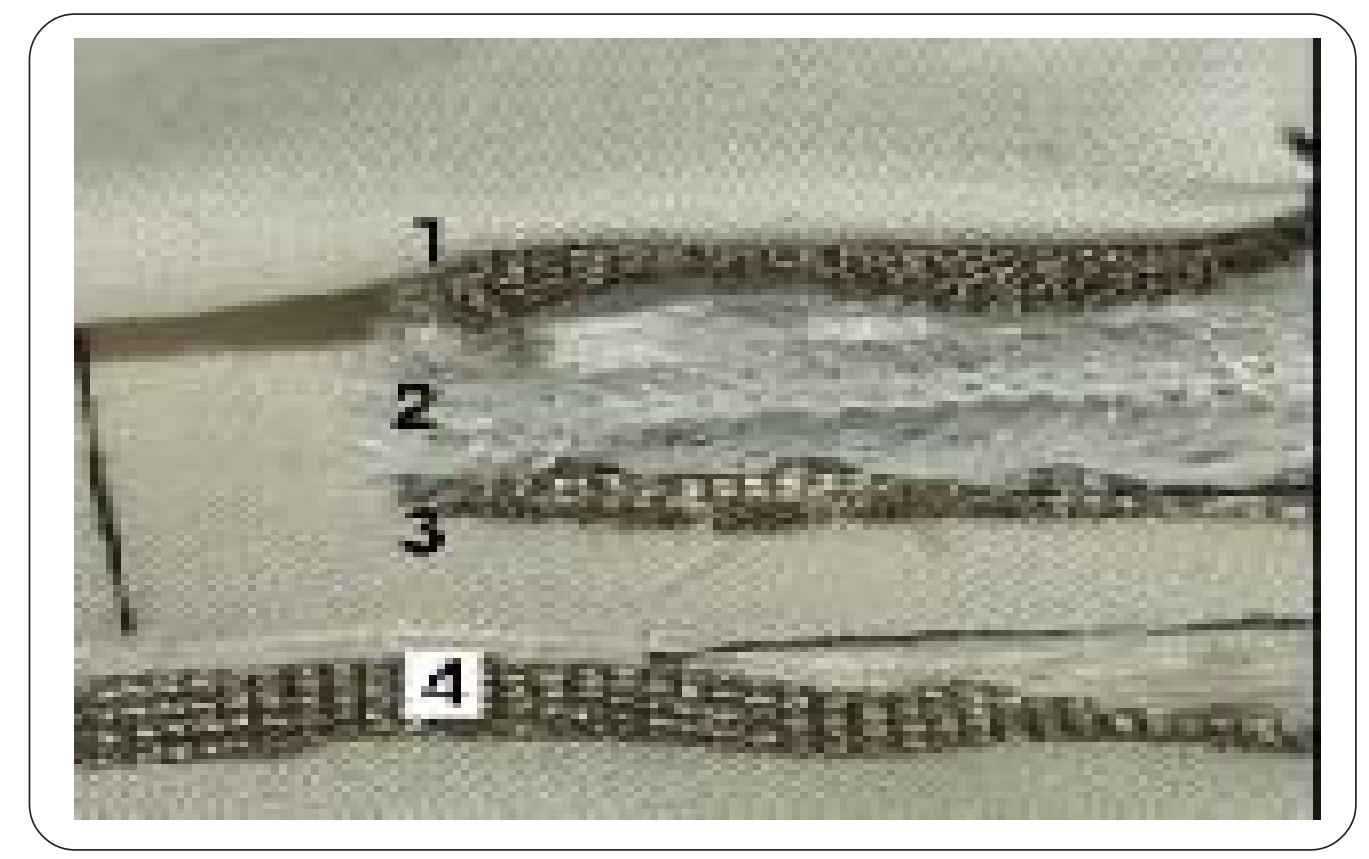

Figure 3. Construction of sensor with one sensing element. 1, 3: Top and bottom electrode of conductive textile, 2: Two layers of compressible spacer, 4: Shielding

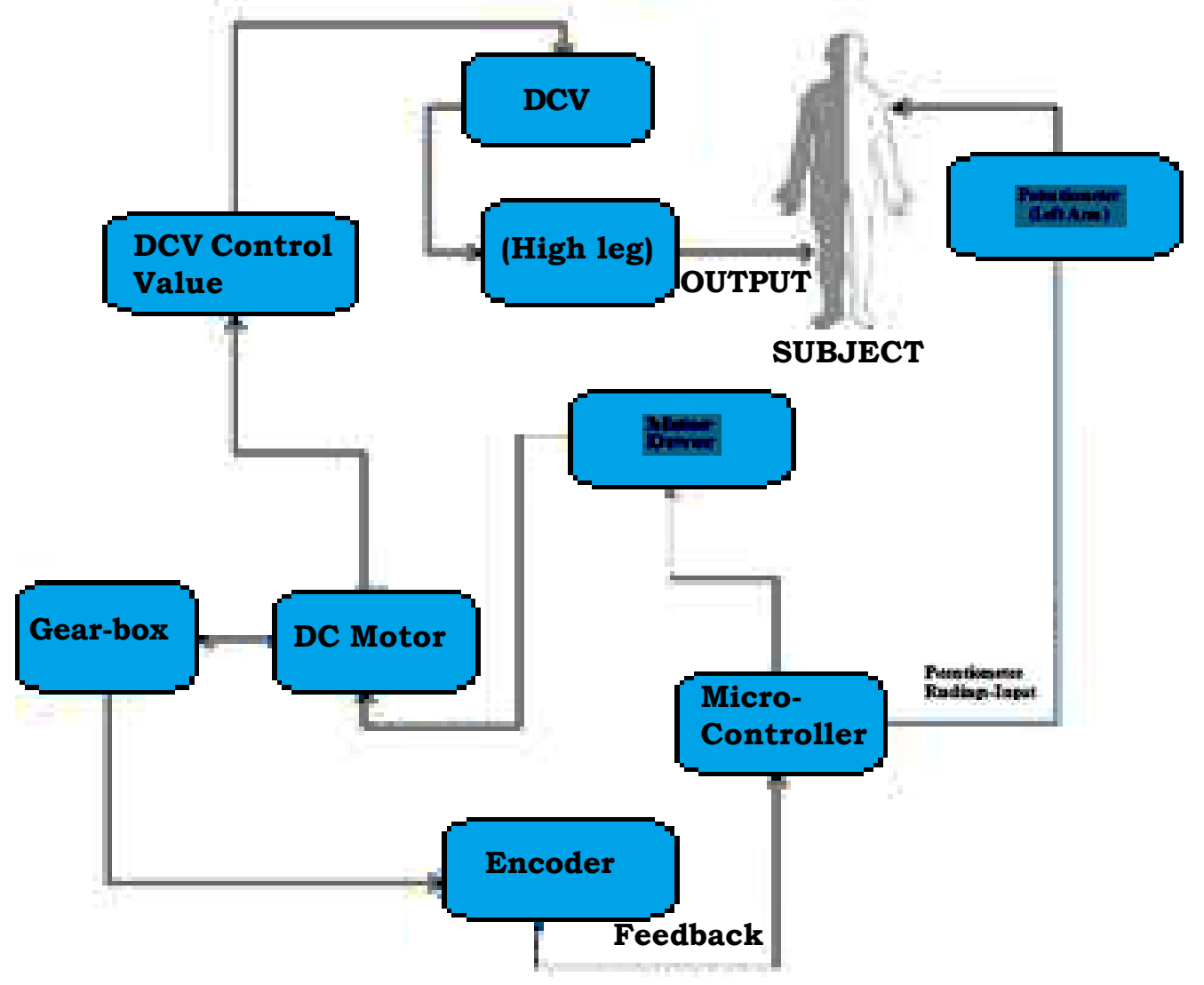

Figure 4. Textile pressure sensor with 16 sensing elements embroidered with conductive yarn 
system. The sensor sends these readings to the Micro-Controller. Immediately the Micro-Controller sends signals to the Solenoid Operated DCV (Directional Control Valve). While it is decreasing its angle, there is a DC Motor which is fixed to the Flow Control Valve is revolving and thereby the stroke length of the piston cylinder is increasing. The speed of the exoskeleton depends on the angle detected by the textile sensors and as a result the control system and generates the output required which triggers the gear box and increases or decreases the speed.

There are two pneumatic actuators one at the backthigh muscle and one at the calf muscle. The back-thigh muscle actuator actuates gradually as soon as it gets information from the sensors. These sensors ensure free flowing movement of the user and does not cause any disruptions for the user. These sensors also ensure comfort for the user.

The role of Encoder is to calculate the amount of rotations performed by the DC Motor and send it to the Micro-Controller to control the motor in opposite directions by the same amount of rotations and both the actuators are retracted once the feet touches the ground by means of a force sensor, which is attached to the rubber sole of the affected feet.

\section{Leg and Thigh Assembly}

\subsection{Leg Assembly}

The Leg Assembly consists of Three Pneumatic actuators namely B, C, D. When actuator B extend whole body frame will not travel in a linear motion due to the rigid links at the top of the body frame which is connected to the base of support of the cylinder and mainly due to low tension spring. As a result, there will be a swing motion which is common in individual's gait cycle. Actuators C, D are always in extended state to support the affected leg from the reaction forces. This allows movement of the subject in any uneven terrain also. Besides there is an elastic strap which facilitates strong binding between the Body frame and the Leg, which is adjustable.

\subsection{Thigh Assembly}

The actuator A is aligned is such a way that the extension stroke of the piston cylinder yields maximum angle, which is responsible for displacement or the stride length. Pneumatic Pressure causes a lot of vibrations if necessary trusses are not given it can even damage the structure. The actuator is connected to the Body frame rigidly and thus its extension/ retraction will cause the thigh muscle to actuate and thereby cause movement. The Body frame is made up of Aluminium considering that it is

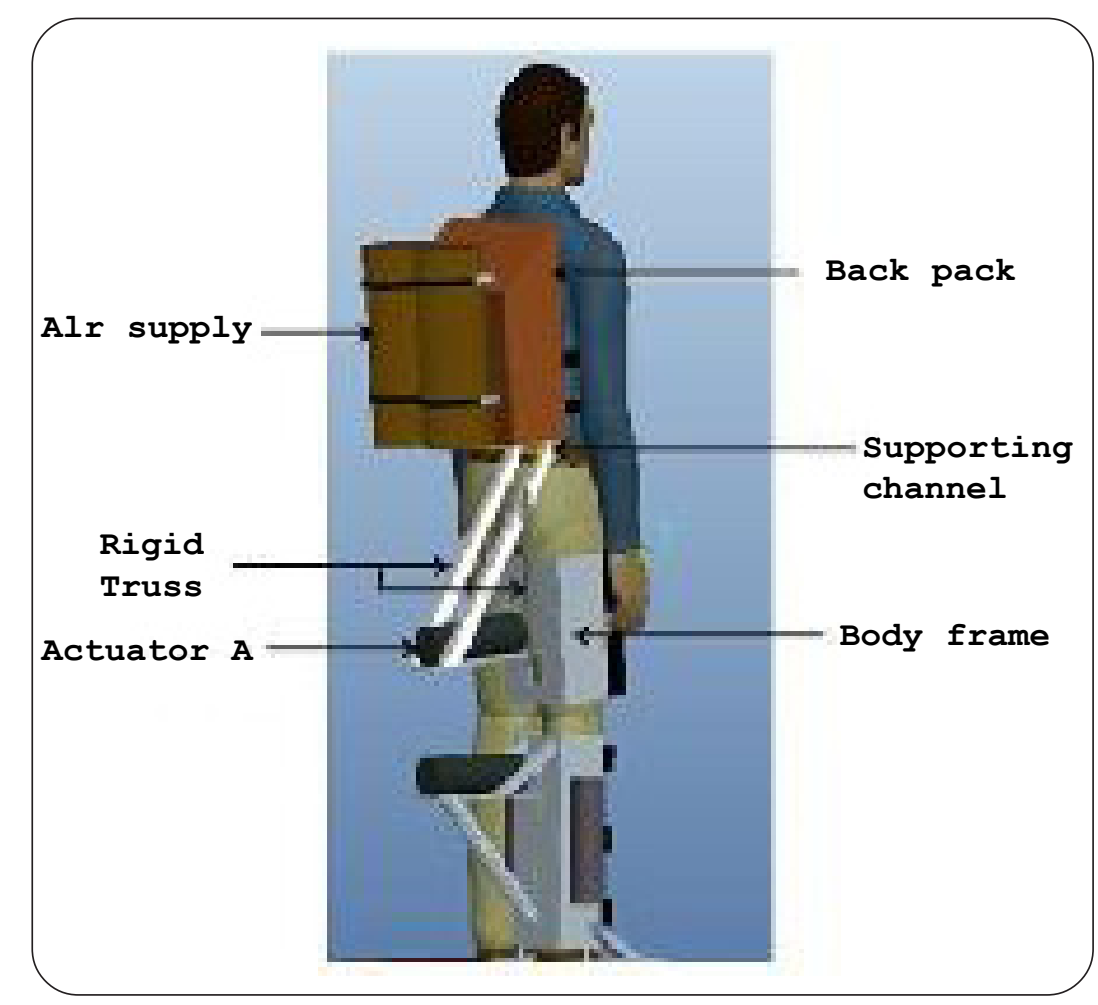




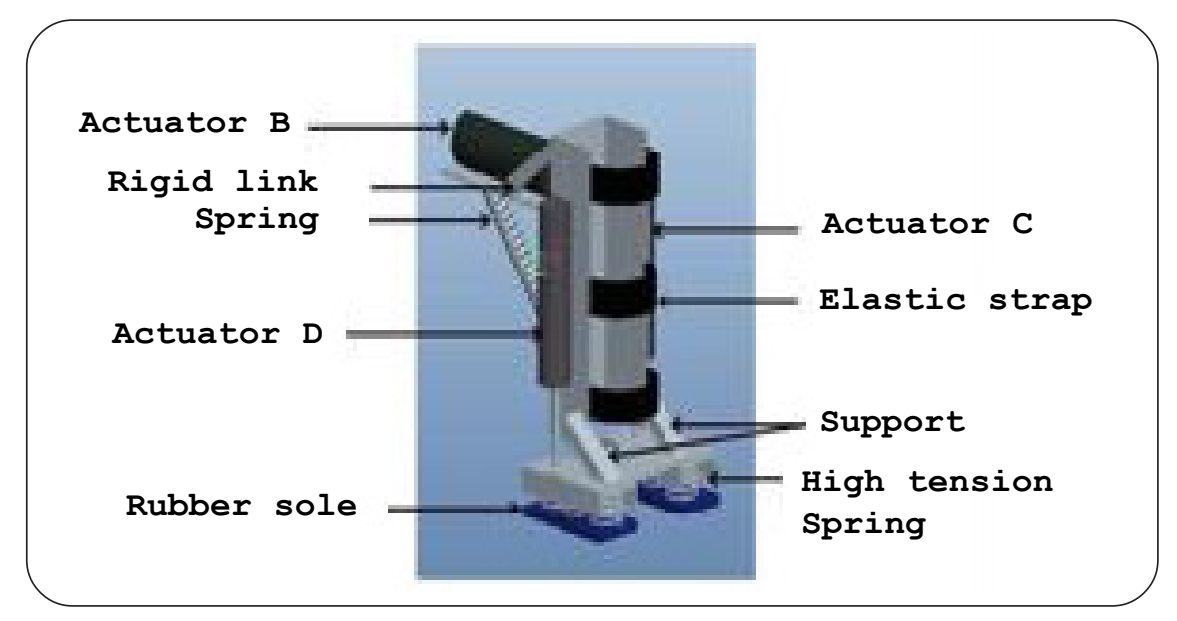

light weight and strong. Thus, there is no direct transmission of force to the thigh muscle which might cause hurt. The inner side of the Body frame is coated with soft sponge material to give cushioning effect. Elastic belts are placed which help fixing the Body frame with the Thigh easily and can be adjusted depending upon the ease of the subject. The Rigid links are welded with the Body Frame thus making it strong.

\section{Conclusion}

Thus, the proposed model can help the people suffering from paraplegia. Paralysation is the ideology is widely explained in this paper. The idea of implementation of a textile sensor in a exoskeleton suit in new and opens new possibilities. Though the concept is in initial phase now, in future much development will be made and this can revolutionize the medical technology and help paralyzed people to walk.

\section{References}

[1] Gui, Lihua., Yang, Zhiyong., Yang, Xiuxia., Gu, Wenjin., Zhang, Yuanshan. (2007). Design and Control Technique Research of Exoskeleton Suit, Proceedings of the IEEE International Conference on Automation and Logistics August 18 - 21, Jinan, China.

[2] http://emedicine.medscape.com/article/320160-overview

[3] http://www.chiro.org/ACAPress/Body_Alignment.html

[4] Varicap series datasheet from MACOM.

[5] Textile Pressure Sensor for Muscle Activity and Motion Detection by Jan Myer, Paul, Gerhard, Austria 